\title{
Improvement of Energy Efficiency in Wireless Sensor Networks Using Low-energy Adaptive Clustering Hierarchy (LEACH)-based Energy Betweenness Model
}

\author{
Yu-Fan Feng, ${ }^{1,2}$ Shu-Guang Pan, ${ }^{1,2}$ Zhi-Yong Huang, ${ }^{3}$ and Hsiung-Cheng Lin ${ }^{4 *}$ \\ ${ }^{1}$ State Key Laboratory of Reliability and Intelligence of Electrical Equipment, \\ Hebei University of Technology, Tianjin, China 300130 \\ ${ }^{2}$ Laboratory of Electromagnetic Field and Electrical Apparatus Reliability of Hebei Province, \\ Hebei University of Technology, Tianjin, China 300130 \\ ${ }^{3}$ China State Construction Eng. Corp. Direction Generale Pour L'Algerie, Algiers, Algeria 16101 \\ ${ }^{4}$ Department of Electronic Engineering, National Chin-Yi University of Technology, Taiwan 41170
}

(Received April 9, 2019; accepted June 26, 2019)

Keywords: sensor network, energy betweenness, protocol of clustering, energy consumption, lifespan of network, balance

The imbalance of energy consumption in wireless sensor networks (WSNs) may affect both network lifetime and reliability. Traditionally, the low-energy adaptive clustering hierarchy (LEACH) protocol has been applied to lower the energy consumption. Although the LEACH protocol can choose cluster heads $(\mathrm{CHs})$ randomly to prevent a number of nodes from premature failure due to overutilization, the discrepancy in the energy distribution under different network topologies may result in a low network performance efficiency. In this paper, the LEACHenergy betweenness (LEACH-EB) model is proposed by taking energy consumption as a constraint condition. It can judge the equilibrium of clustering based on the energy betweenness of each node and realize the optimization of clustering in WSNs. The simulation results verify that the proposed LEACH-EB model can make the clustering more energy-efficient for better performance in terms of reliability and stability than the LEACH protocol. Additionally, the model can significantly reduce the extra energy loss caused by uneven clustering and thus prevent the degradation of network performance from the premature senescence of some nodes.

\section{Introduction}

As the supporting framework of information, networks play an important role such as in applications using the Internet of Things (IoT) in distribution networks. ${ }^{(1)}$ Wireless sensor networks (WSNs) have been recognized as a key enabler for the IoT since its appearance. The extension of the lifetime of WSNs can be ultimately attributed to the study of energy loss based on the evaluation of the number of failed nodes. ${ }^{(2-5)}$ However, in most WSNs, the failure of one or more nodes may not affect regular functions of the whole network. Therefore, an evaluation

*Corresponding author: e-mail: hclin@ncut.edu.tw https://doi.org/10.18494/SAM.2019.2403 
method was proposed to estimate the network lifetime based on functionality, including network connectivity, coverage and quality of service, reliability, balance, and energy consumption. ${ }^{(6,7)}$ For the operating protocol of WSNs, Safia et al. reported a dynamic distributed algorithm that greatly reduces the energy consumption of WSNs in the monitoring process. ${ }^{(8)}$ However, the algorithm is only applicable to mobile wireless networks so the range of application scenarios is limited. In the literature, ${ }^{(9)} \mathrm{RF}$ wireless energy transmission has been applied to WSNs, which solves the problem of the limited energy-carrying capacity of nodes. However, this type of technology still has some challenges in practical applications. Kumar et al. proposed an algorithm by arranging redundant nodes for fence coverage to obtain the optimal number of deployments. ${ }^{(10)}$ It can maximize the overall network lifetime in the case that the life of each node in the network is different. However, deployment optimization and node compensation have certain limitations in dense and complex networks. For irregularly deployed nodes, the probability of the node itself becoming a cluster head $(\mathrm{CH})$ is determined by considering the population of sensing, which is defined as the number of nodes within the sensing range of nodes. As a result, the coverage area of each cluster is almost evenly distributed, reducing the unnecessary repetition of sensing and transmission. ${ }^{(11)}$

Chang et al. proposed a distributed joint optimization routing algorithm based on an analytic hierarchy process (AHP). The remaining energy of the node, the distance from the node to the base station, and the degree of the node were defined as three key decision criteria. ${ }^{(12)}$ In addition, the AHP was used to select the next hop node on the transmitter side to extend the network lifetime. In the literature, ${ }^{(13)}$ a new load-balancing scheme has been improved by applying subnet management to WSNs, which balances the energy consumption of sensor nodes and maximizes the network lifetime through load balancing. Zhang et al. carried out an indepth study on the residual energy of nodes and the energy utilization of data communication links to optimize the topology of WSNs. ${ }^{(14)}$ In addition, Hao et al. proposed the TCAMLPM algorithm to guarantee the functionality and lifetime of a network by using the Markov lifetime prediction model. ${ }^{(15)}$ Energy management is also one of the ways to prolong the lifetime of networks such as applications using forward advanced energy management technology multiple utilization of energy and so forth. ${ }^{(16)}$ For the protocol of WSNs, some scholars such as Ihsan et al. have made comparative studies on a number of protocols in the literature. ${ }^{(17)}$ Heinzelman et al. proposed the low-energy adaptive clustering hierarchy (LEACH) protocol, in which dynamic layering is realized by cycling. Accordingly, the CHs with large energy consumption are shared by all nodes in the operation process. ${ }^{(18)}$ However, this is not conducive to the long-term and stable operation of sensor networks. Kang et al. developed an improved protocol named LEACH-XMP, which introduced the most advanced message transmission method, reducing energy consumption during information transmission in WSNs. ${ }^{(19)}$ The LEACH algorithm has been improved by simplifying the network function and reducing the hardware cost. ${ }^{(20)}$ Under this principle, LEACH-AP achieves fully distributed control and solves the practical limitations of the LEACH algorithm. The advantages and disadvantages of each protocol ${ }^{(18-22)}$ are summarized in Table 1. 
Table 1

Protocols in WSNs.

\begin{tabular}{lcccc}
\hline Protocol & Authors & Features & Advantage & Shortcoming \\
\hline LEACH & $\begin{array}{c}\text { Heinzelman } \\
\text { et al. }\end{array}$ & Turning CHs & $\begin{array}{c}\text { Transfers load } \\
\text { periodically }\end{array}$ & $\begin{array}{c}\text { Probability of nodes } \\
\text { becoming CHs is consistent }\end{array}$ \\
\hline LEACH-AP & Sohn et al. & $\begin{array}{c}\text { More advanced methods } \\
\text { of transmitting messages } \\
\text { between nodes }\end{array}$ & $\begin{array}{c}\text { Reduces energy } \\
\text { consumption } \\
\text { of transmission }\end{array}$ & High cost of technology \\
\hline LEACH-MAC & Batra and Kant & $\begin{array}{c}\text { Controlling number } \\
\text { of CHs }\end{array}$ & Reduces hardware cost & $\begin{array}{c}\text { Harmful for } \\
\text { function of network }\end{array}$ \\
\hline CL-LEACH & $\begin{array}{c}\text { Marappan and } \\
\text { Rodriguez }\end{array}$ & Cross-layer design & $\begin{array}{c}\text { Considers location } \\
\text { information and } \\
\text { residual energy }\end{array}$ & $\begin{array}{c}\text { Computing model is } \\
\text { complex }\end{array}$ \\
\hline
\end{tabular}

\section{LEACH-energy Betweenness (LEACH-EB) Model}

\subsection{Fundamental concept of sensor network}

The monitoring section of a WSN is composed of many wireless sensor nodes (hereinafter referred to as nodes). Each node is composed of three functional modules including sensors, processors, and wireless communication modules. They give the node the ability to detect, receive, and process information in the WSN. Each node is responsible for gathering information to form a complete communication system. All sensor nodes in the monitoring area are responsible for collecting information near themselves and sending it to the designated node, which is called a $\mathrm{CH}$. After the $\mathrm{CH}$ fuses the information it receives, the information is packaged and sent to the base station. CHs form a cluster with all sensor nodes under their jurisdiction, and the detection function of sensor networks is accomplished by several such clusters. The base station is the bridge between the WSN and the user terminal, responsible for the information exchange between the two sides. After the signals are received from the monitoring network, the base station transmits the information to the user terminal by satellite, the Internet, and so on. In addition, the base station also broadcasts the instructions transmitted by the user terminal to each sensor node. In practical applications, the size of the node itself and its carrying energy are limited, which have become key factors affecting the stable operation of networks. The information transfer stream is shown in Fig. 1.

\subsection{Development of the LEACH-EB model}

The proposed LEACH-EB model is divided into four stages, as shown in Fig. 2.

Step 1: Input basic data

After the basic information in LEACH-EB is determined, the $\mathrm{CH}$ is preselected and the energy median of each node in the precluster is calculated.

Step 2: Form possible clusters

The variance of the energy betweenness between nodes is compared and stored in a variable. 


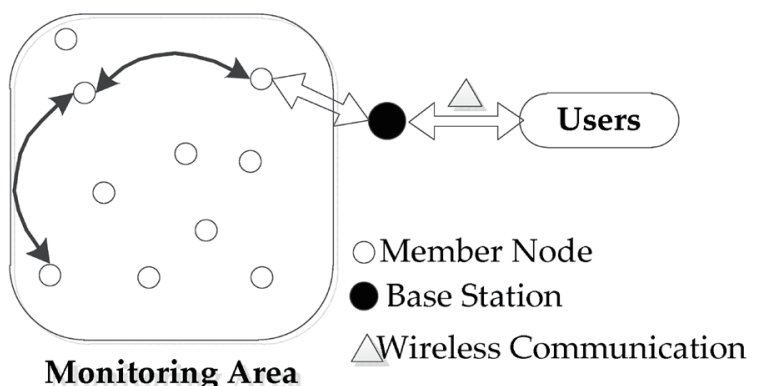

Fig. 1. Typical sensor network architecture.

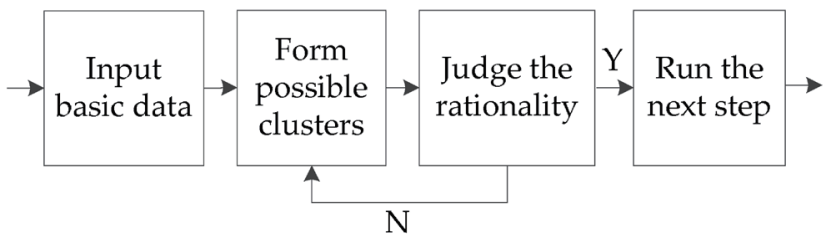

Fig. 2. Flowchart of the LEACH-EB model.

Step 3: Judge the rationality

After the preselection for a specified number of times, the cluster with the smallest variance of the energy betweenness of nodes is selected to accomplish the final running.

Step 4: Run the next step

After completing a round, the cluster selection is carried out again by the same procedure.

\subsubsection{Energy model of $\mathrm{CH}$}

The energy required by the $\mathrm{CH}$ is used to receive messages from each node, aggregate the received messages, and transmit the aggregated messages to the base station, corresponding to Eqs. (1)-(3), respectively:

$$
\begin{gathered}
E_{C H 1}(c)=l E_{\text {elec }}\left(\frac{N}{k}-1\right), \\
E_{C H 2}(c)=l E_{D A} \frac{N}{k}, \\
E_{C H 3}(c)=l E_{\text {elec }}+l \varepsilon_{m p} d_{t o B S}^{4},
\end{gathered}
$$

where $l$ represents the length (bits) of messages; $E_{\text {elec }}$ in Eqs. (1) and (3) denotes the energy consumption for receiving and transmitting messages, respectively. $N$ represents the total number of sensor nodes; $k$ represents the number of clusters; $E_{D A}$ represents the energy consumption of single-unit data fusion; $\varepsilon_{m p}$ is the magnification factor of the multipath transmission model; $d_{t o B S}$ is the distance from the $\mathrm{CH}$ to the base station. The number of nodes in the cluster is expressed by the average $N / k$. The total energy consumption of the $\mathrm{CH}$ is the sum of Eqs. (1)-(3), defined as

$$
E_{C H}(c)=E_{C H 1}(c)+E_{C H 2}(c)+E_{C H 3}(c) .
$$




\subsubsection{Energy model of general nodes}

In one round, a node only sends a message to the $\mathrm{CH}$ once, and its transmitting distance is relatively short. Therefore, the power loss adopts a free-space model in which there is only one clear line-of-sight path between the transmitter and the receiver. The energy consumption of node $n$ in one round of operation is expressed as

$$
E_{N O N}(n)=l E_{\text {elec }}+l \varepsilon_{s f} d_{\text {toCH }}^{2},
$$

where the magnification factor of the model is $\varepsilon_{s f}$ and the distance from the node to the $\mathrm{CH}$ is denoted as $d_{t o C H}$. Note that the final data of this round will continue to be computed as the input data for the next round until most of the nodes are too exhausted to operate the network functions.

\subsubsection{Energy betweenness}

Betweenness is defined as the ratio of the number of shortest paths passing through one node to the total number of shortest paths in the network, first proposed by Bavelas, ${ }^{(23)}$ and its formula is

$$
B C(i)=\sum_{i \neq s, i \neq e, s \neq e} \frac{l_{s e}^{i}}{l_{s e}}
$$

where $l_{s e}$ is the shortest path from node $e$ as the end point to node $s$ as the starting point in the network, and $l_{s e}^{i}$ is the shortest path through node $i$ among all paths.

The energy betweenness of a node is based on the energy consumption and used to quantitatively evaluate the importance of nodes in sensor networks. A node with a large energy betweenness consumes a lot of energy in the network, that is, it has a large load to carry out a heavy task in the running of the network. Such nodes are prone to failure due to energy exhaustion. Therefore, the energy betweenness used to evaluate the network performance is defined as

$$
E_{B C}(i)=\sum_{i \neq s, i \neq e, s \neq e} \frac{E_{s e}^{i}}{E_{s e}},
$$

where $E_{s e}$ is the energy consumed by all nodes in the sensor network after the end of one round and $E_{s e}^{i}$ is the energy consumed by node $i$ in this round.

The total energy consumed by the network is written as

$$
E_{s e}=\sum E_{C H}(c)+\sum E_{N O N}(n)
$$


Depending on the task of the node, $E_{s e}^{i}$ is calculated as

$$
\left\{\begin{array}{c}
E_{s e}^{i}=E_{C H}(c), i=c \\
E_{s e}^{i}=E_{N O N}(n), i=n
\end{array}\right.
$$

where $c$ depicts a $\mathrm{CH}$ node and $n$ represents a common node.

After the energy betweenness of each node is obtained in each round, the clustering is determined by the following equation as the target

$$
\min \sigma(j)=\frac{1}{n} \sum_{k=1}^{n}\left(\bar{E}_{B C}-E_{B C}(i)\right)^{2}
$$

where $n$ is the total number of nodes in the sensor network, $\bar{E}_{B C}$ is the average of the energy betweenness of all nodes, $E_{B C}(i)$ is the energy betweenness of the ith node, and $\sigma$ is the variance of the energy betweenness. The least value of $\sigma$ among the clusters is the optional clustering variance.

\subsubsection{Clustering strategy based on the energy betweenness}

The establishment of a cluster in the LEACH protocol may make the $\mathrm{CH}$ distribution nonuniform owing to the $\mathrm{CH}$ selection with a random rotation. To achieve reasonable clustering, the energy betweenness is newly employed to perform the process of $\mathrm{CH}$ selection, as shown in Fig. 3.

Firstly, the basic parameters are initialized, including the number of rounds, $r$; the number of nodes, $n$, participating in the cluster selection; the maximum number of cluster selections, $M$, in each round; the basic information of each node; and the parameters of the network energy loss. $j$ is set from 0 to $M$, and $i$ is set from 1 to $N$. When $j-1$ reaches 0 , the variance is taken as

$$
\sigma(0)=10
$$

After the initialization of the basic data, the first trial clustering in this round, i.e., $j=1$, is started. Each node is assigned a random value from 0 to 1 , which is stored as $T_{i}$. A possible clustering is then formed ${ }^{(4)}$ by comparing the magnitudes of $T_{i}$ and $P_{i}$. $P_{i}$ is expressed as

$$
P_{i}=\frac{k}{n-k \times\left(r \bmod \frac{n}{k}\right)},
$$

where $r$ denotes the number of rounds at this time, $k$ denotes the expected number of $\mathrm{CHs}$ in a particular round, and $n$ denotes the total number of nodes in the cycles. $P_{i}$ is for a node that has served as a $\mathrm{CH}$ before the number of rounds $r$ is 0 . 


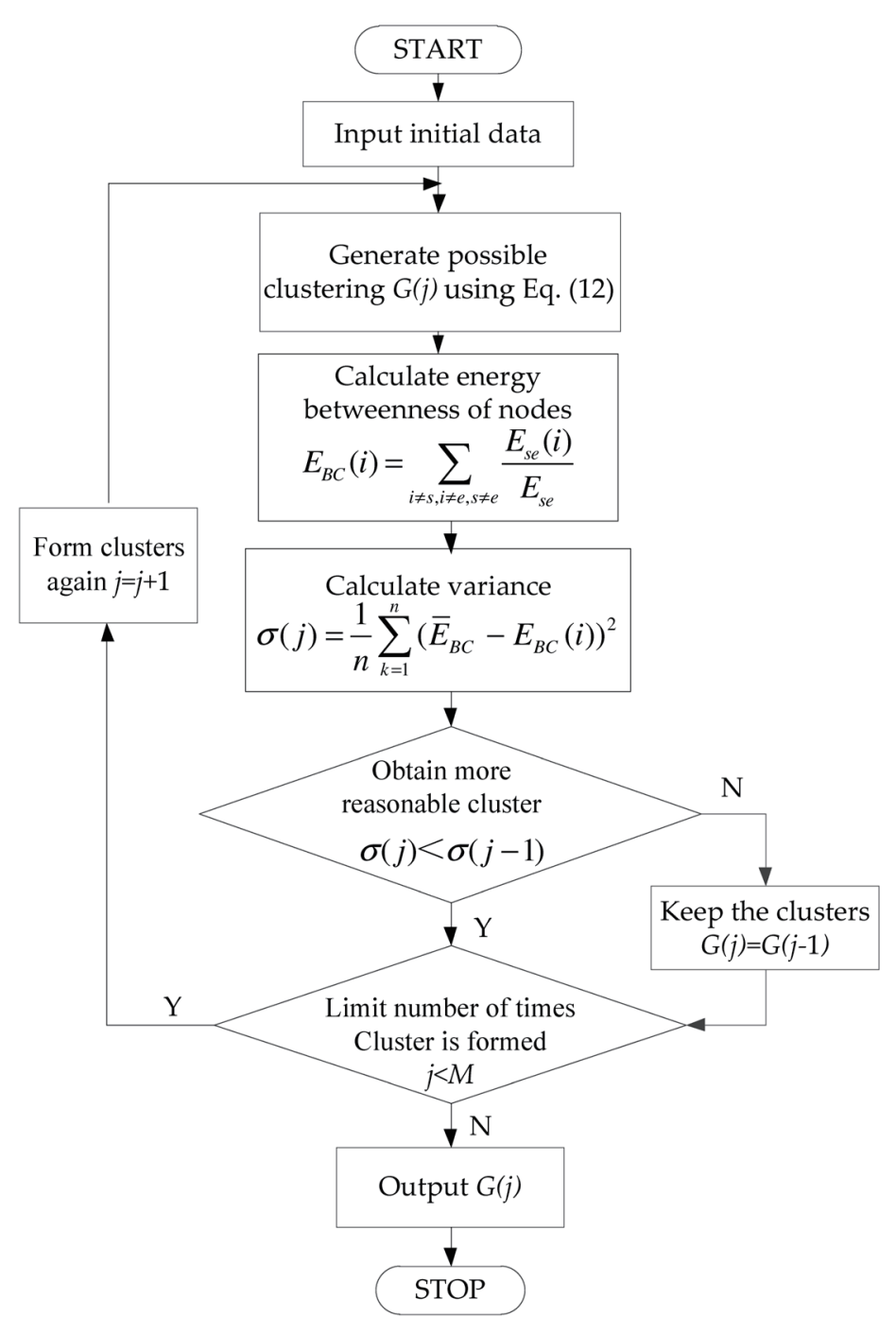

Fig. 3. Flowchart of $\mathrm{CH}$ selection.

Then, the energy betweenness and the variance of the energy betweenness of each node in this clustering are calculated using Eqs. (7) and (10). This variance is compared with that obtained by $j-1$ clustering, and the clustering data with the smaller variance is stored in $G(j)$. If $j$ is less than the maximum number of clusters $M$, then

$$
j=j+1 .
$$

The initial state is used to select the cluster again, and the variance in the new cluster is compared with the last one until $j=M$. Finally, the clustering $G(j)$ with the minimum variance of energy betweenness within several cycles is obtained to accomplish information collection and transmission in sensor networks. 


\section{Results and Analysis}

\subsection{Parameter selection in the model performance}

The parameters chosen for the sensor networks and the energy model used in the proposed algorithm are listed in Tables 2 and 3, respectively.

\subsection{Performance results}

In the LEACH protocol, $\mathrm{CHs}$ tend to be concentrated in a certain area, whereas the $\mathrm{CHs}$ of LEACH-EB are more equally distributed as a result of the optimization of the cluster selection, as shown in Fig. 4. Some nodes in the network may fail after a certain time. As can be seen, the distribution of CHs shown in Fig. 4(a) is concentrated on the right side, resulting in the failed nodes being concentrated on the right side. This inevitably weakens the validity of data detection because the data sources are over concentrated on one side, which is not conducive to the final data analysis. In Fig. 4(b), the CHs and failed nodes when using the LEACH-EB model are scattered more uniformly, making the data measured by sensor networks more stable and reliable.

The membership of clusters is shown in Fig. 5, where the blue and red lines represent the clustering situations for the LEACH protocol and LEACH-EB model, respectively. For the LEACH protocol, it is found that the number of members in each cluster fluctuates, reaching twice the average value. On the other hand, the number of cluster members of the LEACH-EB model is more uniform on average without significant fluctuation.

Figure 6 shows how the number of failed nodes increases with the number of rounds. The cumulative number of failed node over 1000 rounds is shown in Fig. 6. It reveals that the node failure time is longer and the failure rate is lower for the LEACH-EB model than for the LEACH protocol. For example, the first node failure when using the LEACH protocol appears at the 159th round, and the number of failed node is 96 . On the other hand, for the LEACH-EB model, the first node failure appears at the 220th round and the total number of failed nodes is only 85 . The lifetime of sensor networks when using the LEACH-EB model is extended by $38.36 \%$, as determined from the comparison of the first node failure time. Moreover, the number of failed nodes is reduced by about $11 \%$ for the LEACH-EB model.

Table 2

Specification of sensors networks.

\begin{tabular}{lc}
\hline Parameters & Value \\
\hline Number of starting nodes, $N$ & 100 \\
Scope of network area, $S$ & $100 \times 100$ \\
Base station location, $(X, Y)$ & $(50,50)$ \\
Packet length, $D M$ & $4000 \mathrm{bits}$ \\
Control data length, $C M$ & $32 \mathrm{bits}$ \\
Proportion of CHs, $k / N$ & 0.1 \\
Number of rounds, $r$ & 1000 \\
Original energy, $E$ & $0.02 \mathrm{~J}$ \\
Maximum number of clusters per round, $M$ & 10 \\
\hline
\end{tabular}

Table 3

Energy model parameters.

\begin{tabular}{lc}
\hline Parameters & Value \\
\hline Receive/emission loss, $E_{\text {elec }}$ & $50 \mathrm{~nJ} / \mathrm{bit}$ \\
Loss of data fusion, $E_{D A}$ & $5 \mathrm{~nJ} / \mathrm{bit} / \mathrm{signal}$ \\
Parameter of free-space model, $\varepsilon_{s f}$ & $10 \mathrm{pJ} / \mathrm{bit} / \mathrm{m}^{2}$ \\
Parameter of multipath & \\
transmission model, $\varepsilon_{m p}$ & $0.0013 \mathrm{pJ} / \mathrm{bit} / \mathrm{m}^{4}$ \\
\hline
\end{tabular}




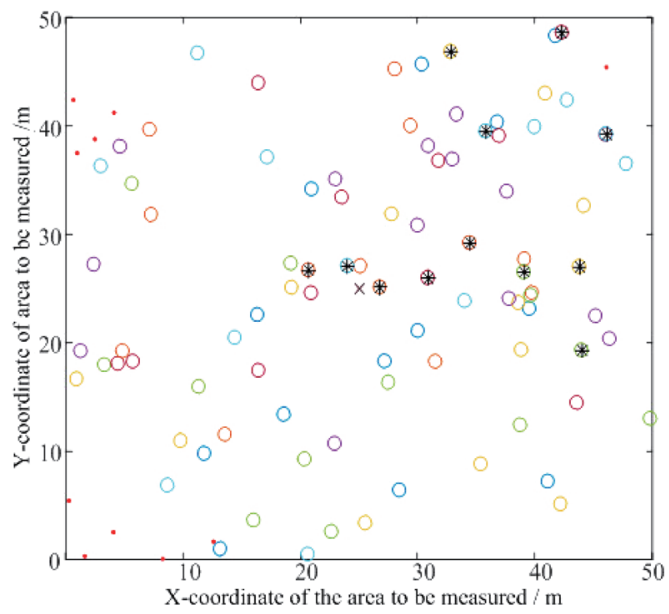

(a)

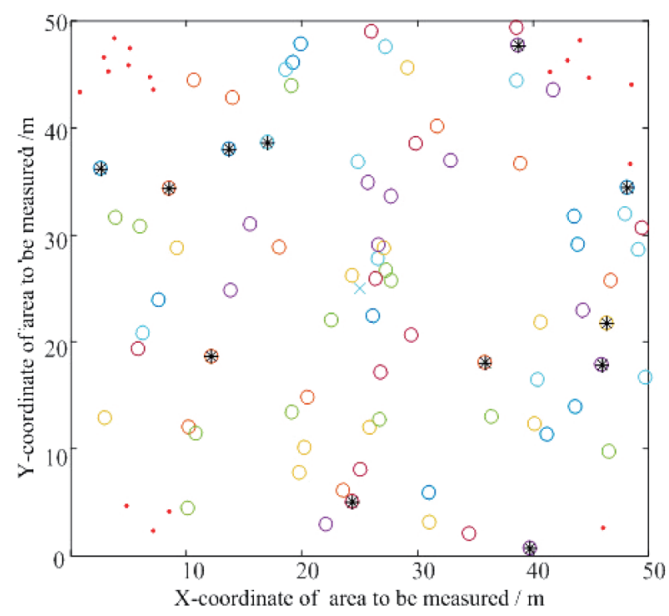

(b)

Fig. 4. (Color online) Distributions of CHs: (a) LEACH protocol and (b) LEACH-EB model.

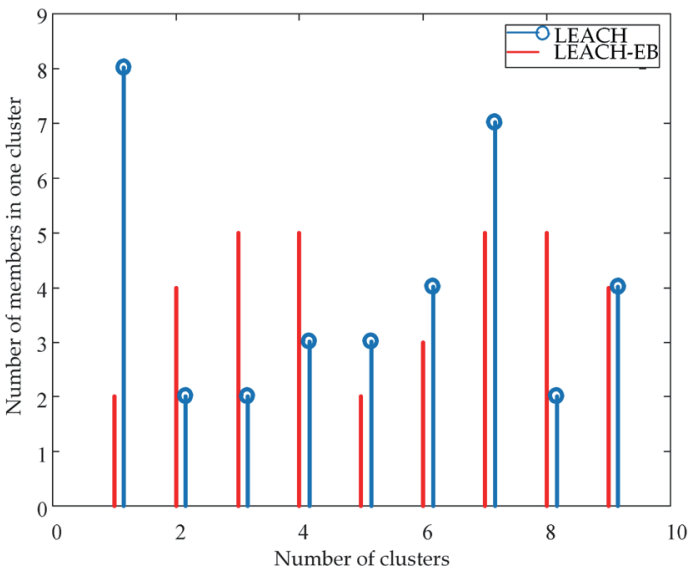

Fig. 5. (Color online) Membership of clusters.

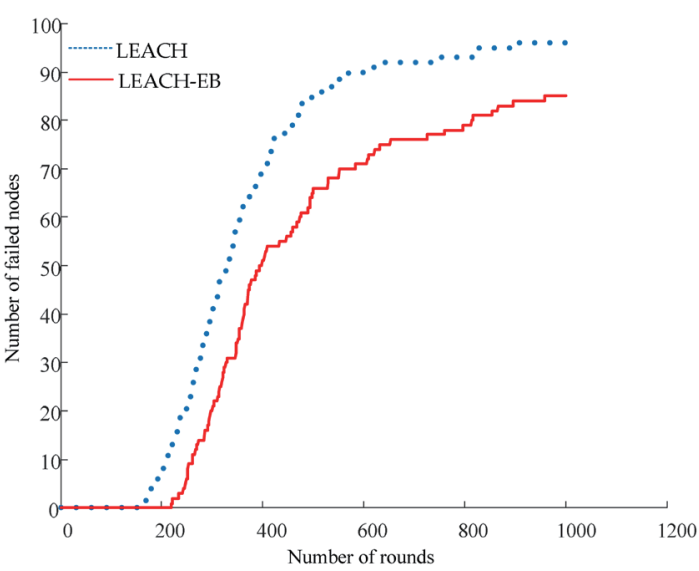

Fig. 6. (Color online) Number of failed nodes in the network.

A comparison of the remaining energy of each node between the LEACH protocol and the LEACH-EB model under the same conditions is shown in Fig. 7. Note that a node fails when its residual energy is zero or negative because its energy has been exhausted. On the basis of this principle, as can be seen from Fig. 7, the total ratio of nodes with the positive residual energy for the LEACH-EB model is clearly higher than that for the LEACH protocol. This means that the LEACH-EB model can consume less energy and thus improve the network performance efficiency. The number of failed nodes over 500 rounds observed using the LEACH and LEACH-EB models is shown in Fig. 8.

It is clear that the LEACH-EB model is superior to the LEACH protocol in the terms of the number of failed nodes in either 50-, 100-, and 200-node networks over 500 rounds, as shown in Figs. 8(a)-8(c), respectively. This indicates that the number of failed nodes when using the 


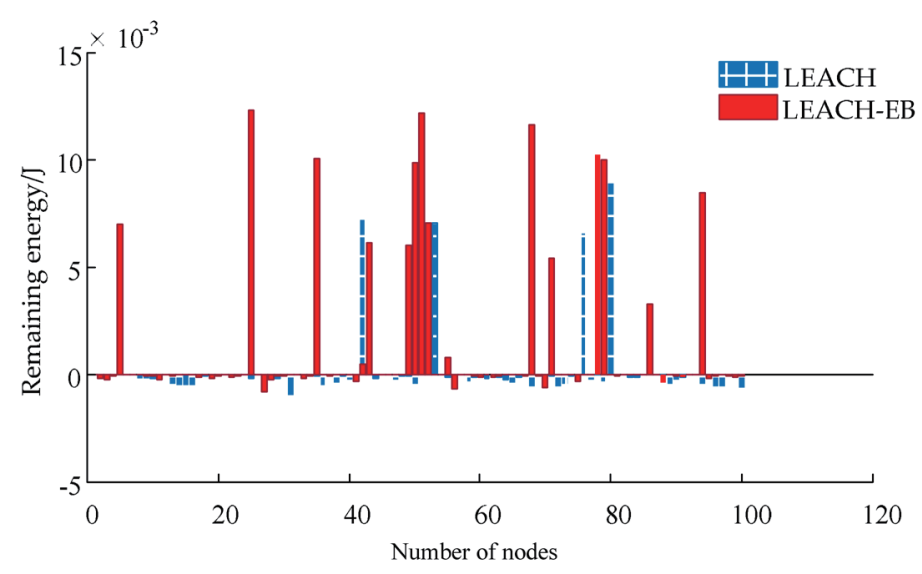

Fig. 7. (Color online) Comparison of remaining energy.

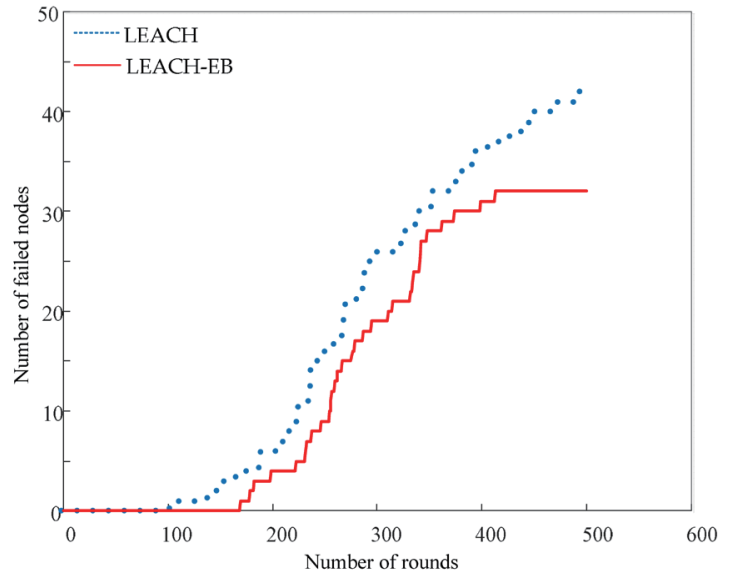

(a)

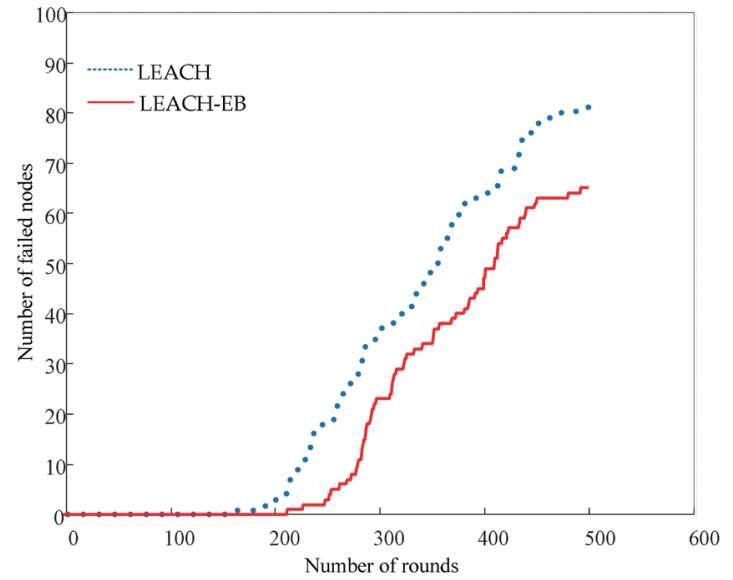

(b)

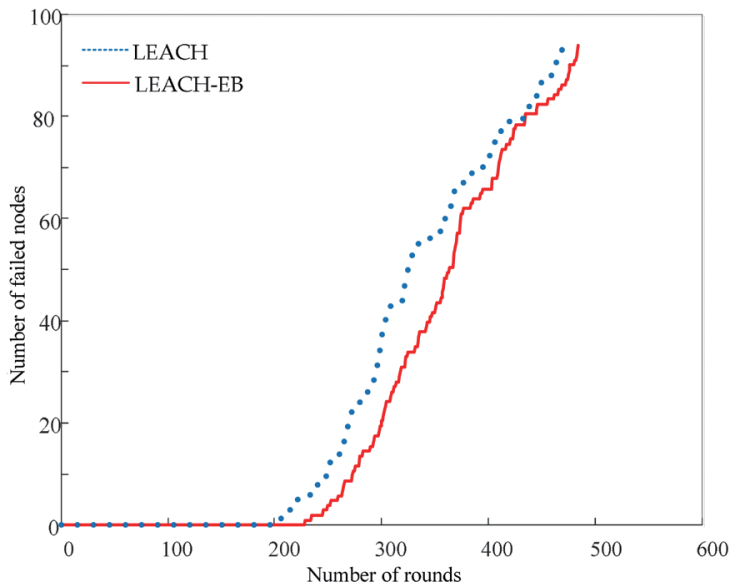

(c)

Fig. 8. (Color online) Number of failed nodes in different networks in the case of (a) 50, (b) 100, and (c) 200 nodes. 
LEACH-EB model is reduced considerably, and the number of failed nodes in each round is less than that for the LEACH protocol. After completing the same number of tasks, the sensor network running with the LEACH-EB model will have more surviving nodes and require less energy to operate. However, increasing the number of nodes causes more nodes to fail for both methods, resulting in the steeper curves of the number of failed nodes plotted against the number of rounds. In other words, the energy loss caused by the uncertainty of the number of $\mathrm{CHs}$ is thus increased.

\section{Conclusions}

Balanced clustering in sensor networks plays a key role in improving both network lifetime and reliability. The LEACH-EB model proposed in this study has a constraint on the minimum variance of energy betweenness to successfully achieve balanced clustering in sensor networks. The main contribution of this paper are summarized as follows.

1. On the basis of LEACH, the variance of energy betweenness is minimized, the energy consumption of each node is balanced, and the $\mathrm{CH}$ selection and clustering algorithm are further optimized.

2. The $\mathrm{CH}$ distribution is made more uniform and the difference in the number of members between clusters is also reduced. This is conducive to balancing the energy consumption for each node during the network operation, thus maintaining the network energy balance.

3. The problem of uneven clustering in sensor networks is resolved using the energy betweenness algorithm. The number of failed nodes decreases for the same number of rounds, thus effectively prolonging the working time of WSNs.

4. The LEACH-EB model is beneficial to the energy distribution of sensor nodes and makes the utilization of nodes energies more reasonable and efficient.

We have demonstrated that the proposed minimum variance of energy betweenness improved the distribution of $\mathrm{CHs}$ and the efficiency of node clustering. The number of failed nodes when using the LEACH-EB model in each round was less than that for the LEACH protocol. Therefore, the energy loss of sensor networks can be balanced effectively, thus saving network energy. Moreover, the uneven energy consumption in WSNs can also be reduced via the appropriate selection of CHs.

\section{Acknowledgments}

This research was funded by National Natural Science Foundation of China and Natural Science Foundation of Hebei Province of China.

\section{Conflicts of Interest}

The authors declare no conflicts of interest. The funders had no role in the design of the study; in the collection, analyses, or interpretation of data; in the writing of the manuscript, or in the decision to publish the results. 


\section{References}

1 T. Yang, Z. Huang, H. Pen, and Y. Zhang: J. Sens. 2017 (2017) 9303989.

2 L. L. Li, X. B. Zhang, M. L. Tseng, L. Ming, and Y. Han: J. Clean Prod. 185 (2018) 198.

3 C. Wang, W. Shi, X. Wang, X. Jiang, Y. Yang, L. Wei, and Z. Ling: Appl. Energy 187 (2017) 10.

4 E. Iturriaga, U. Aldasoro, A. Campos-Celador, and J. M. Sala: Energy 138 (2017) 954.

5 I. Dietrich and F. Dressler: ACM Trans. Sens. Netw. 5 (2009) 1.

6 A. F. Liu, P. H. Zhang, and Z. G. Chen: J. Parallel Distrib. Comput. 71 (2011) 1327.

7 L. L. Li, C. M. Lv, M. L. Tseng, and J. Sun: Appl Soft Comput. 65 (2018) 69.

8 A. A. Safia, Z. A. Aghbari, and I. Kamel: J. Netw. Syst. Manag. 24 (2016) 92.

9 S. Bi, C. K. Ho, and Z. Rui: IEEE Commun. Mag. 53 (2014) 117.

10 S. Kumar, T. H. Lai, M. E. Posner, and P. Sinha: IEEE Trans. Mob. Comput. 9 (2018) 1161.

11 H. Shin, S. Moh, I. Chung, and M. Kang: Wireless Pers. Commun. 82 (2015) 995.

12 Y. Chang, H. Tang, B. Li, and X. Yuan: IEEE Commun. Lett. 99 (2017) 2718.

13 H. Y. Kim: Cluster Comput. 19 (2016) 279.

14 W. Zhang, Y. He, M. Wan, M. Kumar, and T. Qiu: EURASIP J. Wireless Commun. 2018 (2018) 292.

15 X. Hao, L. Wang, Y. Ning, D. Geng, and C. Bai: Ad Hoc Netw. 78 (2018) 13.

16 L. L. Li, X. D. Chen, M. L. Tseng, C. H. Wang, K. J. Wu, and K. L. Ming: J. Clean Prod. 166 (2017) 844.

17 A. Ihsan, K. Saghar, T. Fatima, and O. Hasan: Comp. Stand. Inter. 62 (2018) 119.

18 W. B. Heinzelman, A. P. Chandrakasan, and H. Balakrishnan: IEEE T. Wireless Commun. 1 (2002) 660.

19 J. Kang, I. Sohn, and S. H. Lee: Sensors 19 (2018) 75.

20 I. Sohn, J. Lee, and S. H. Lee: IEEE Commun. Lett. 20 (2016) 558.

21 P. K. Batra and K. Kant: Wireless Netw. 22 (2016) 49.

22 P. Marappan and P. Rodrigues: Wireless Netw. 22 (2015) 1415.

23 A. Bavelas: Hum. Organ. 7 (1948) 16. 